\title{
Immunogenicity of a first dose of mRNA- or vector-based SARS-CoV-2 vaccination in dialysis patients: a multicenter prospective observational pilot study
}

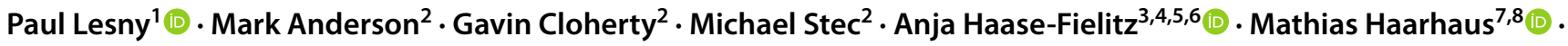 \\ Carla Santos $^{7,9} \cdot$ Carlos Lucas $^{7} \cdot$ Fernando Macario $^{7} \cdot$ Michael Haase ${ }^{1,7,10}$ (i)
}

Received: 10 May 2021 / Accepted: 18 May 2021 / Published online: 29 May 2021

(c) The Author(s) 2021

\begin{abstract}
Background Dialysis patients are at risk for lower SARS-CoV-2-vaccine immunogenicity than the normal population. We assessed immunogenicity to a first mRNA- or vector-based SARS-CoV-2-vaccination dose in dialysis patients.

Methods In a multicenter observational pilot study, 2 weeks after a first vaccination (BNT162b2/Pfizer-BioNTech [Comirnaty] or ChAdOx1 nCoV-19/Oxford-Astra-Zeneca [Vaxzevria]), hemodialysis patients ( $\mathrm{N}=23)$, peritoneal dialysis patients $(\mathrm{N}=4)$ and healthy staff $(\mathrm{N}=14)$ were tested for SARS-CoV-2-spike IgG/IgM, Nucleocapsid-protein-IgG-antibodies and plasma ACE2-receptor-binding-inhibition capacity. Hemodialysis patients who had had prior COVID-19 infection $(\mathrm{N}=18)$ served as controls. Both response to first SARS-CoV-2 vaccination and IgG spike-positivity following prior COVID-19 infection were defined as SARS-CoV-2 spike IgG levels $\geq 50 \mathrm{AU} / \mathrm{mL}$.

Results Vaccination responder rates were $17.4 \%$ (4/23) in hemodialysis patients, $100 \%$ (4/4) in peritoneal dialysis patients and $57.1 \%$ (8/14) in staff (HD vs. PD: $p=0.004$, HD vs. staff: $p=0.027$ ). Among hemodialysis patients, type of vaccine (Comirnaty $\mathrm{N}=11$, Vaxzevria $\mathrm{N}=12,2$ responders each) did not appear to influence antibody levels (IgG spike: Comirnaty median 0.0 [1.-3. quartile 0.0-3.8] versus Vaxzevria 4.3 [1.6-20.1] AU/mL, p=0.079). Of responders to the first dose of
\end{abstract}

Michael Haase

michael.haase@diaverum.com

Diaverum Renal Care Center, 14469 Potsdam, Germany

2 Abbott Infectious Disease Research, Chicago, IL 60064-3500, USA

3 Brandenburg Medical School Theodor Fontane, 16816 Neuruppin, Germany

4 Faculty of Health Sciences Brandenburg, 14469 Potsdam, Germany

5 Institute of Integrated Health Care Systems Research \& Social Medicine, Otto-von-Guericke-University Magdeburg, 39120 Magdeburg, Germany

6 Department of Cardiology, Brandenburg Heart Center, Immanuel Hospital, 16321 Bernau, Germany

7 Diaverum AB, 21532 Malmö, Sweden

8 Division of Renal Medicine, Department of Clinical Sciences, Intervention and Technology, Karolinska Institutet, Karolinska University Hospital, 17177 Stockholm, Sweden

9 Cardiovascular Research and Development Unit, Faculty of Medicine, 4200-319 Porto, Portugal

10 Medical Faculty, Otto-von-Guericke University Magdeburg, Leipziger Str. 44, 39120 Magdeburg, Germany 
SARS-CoV-2 vaccination among hemodialysis patients $(\mathrm{N}=4 / 23)$, median IgG spike levels and ACE2-receptor-bindinginhibition capacity were lower than that of IgG spike-positive hemodialysis patients with prior COVID-19 infection (13/18, 72.2\%): IgG spike: median 222.0, 1.-3. quartile 104.1-721.9 versus median 3794.6, 1.-3. quartile 793.4-9357.9 AU/mL, $\mathrm{p}=0.015$; ACE2-receptor-binding-inhibition capacity: median 11.5\%, 1.-3. quartile 5.0-27.3 versus median 74.8\%, 1.-3. quartile 44.9-98.1, $\mathrm{p}=0.002$.

Conclusions Two weeks after their first mRNA- or vector-based SARS-CoV-2 vaccination, hemodialysis patients demonstrated lower antibody-related response than peritoneal dialysis patients and healthy staff or unvaccinated hemodialysis patients following prior COVID-19 infection.

\section{Graphic abstract}

Immunogenicity of a $1^{\text {st }}$ dose of mRNA- or vector-based SARS-Cov-2 vaccination in dialysis patients

$1^{\text {st }}$ vaccination dose $\quad 2$ weeks
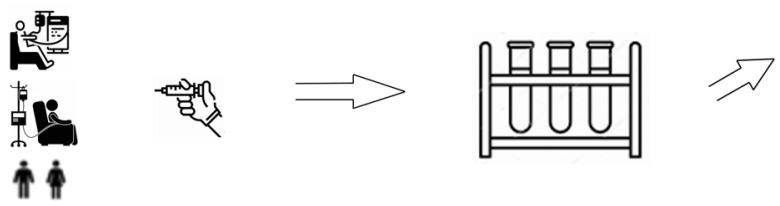

Vaccinated participants

- 23 hemodialysis patients

- 4 peritoneal patients

- 14 healthy staff

Participants after COVID-19

- 18 hemodialysis patients
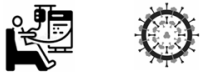

\section{Conclusion}

Most hemodialysis patients and a considerable proportion of healthy staff exhibited an insufficient antibody-related response two weeks after a first mRNA- or vector-based SARS-CoV-2 vaccination, indicating the need to continue with protective measures.

- IgG and IgM spike

- IgG Nucleocapsid

- ACE2 receptor binding inhibition

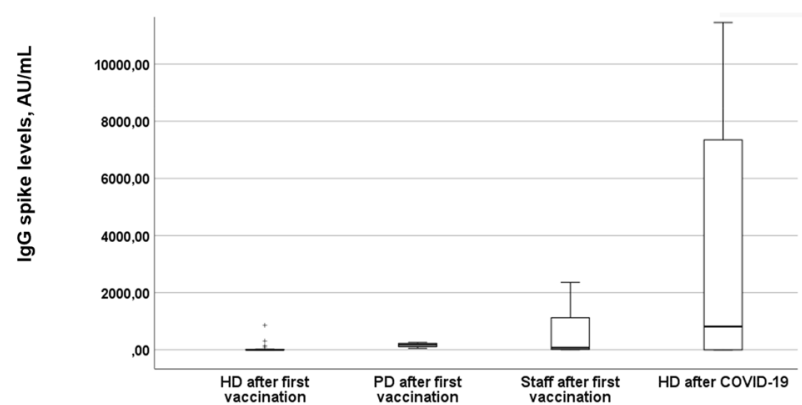

Findings

After first mRNA- or vector-based vaccine dose, responder rates, antibody levels and neutralizing capacity were low in hemodialysis patients and staff.

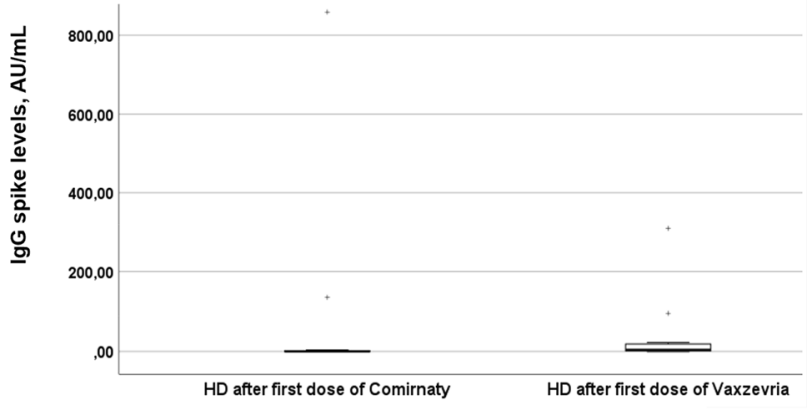

Keywords mRNA- or vector-based SARS-CoV-2 vaccination $\cdot$ Responder $\cdot$ Hemodialysis $\cdot$ Peritoneal dialysis $\cdot$ COVID$19 \cdot$ ACE2 receptor binding inhibition capacity

\section{Introduction}

Several two-dose SARS-CoV-2 vaccines have been approved to prevent COVID-19 infection, with a reported vaccine efficacy of $90-95 \%$ in the normal population after the second dose. Dialysis patients are at high risk for COVID-19 infection and mortality $[1,2]$ but were not included in the vaccination registration trials. Small observational studies showed a sufficient immune response several weeks after the second SARS-CoV-2 mRNA-based vaccination [3, 4]. However, no study compared the effect of a first mRNA-based vaccination with that of a vector-based vaccination on antibody response in hemodialysis patients.

In a pilot study, we aimed to assess the antibody-related immunogenicity of a first dose of mRNA- or vector-based
SARS-CoV-2 vaccine in this patient population compared to that of peritoneal dialysis patients, staff and unvaccinated hemodialysis patients who had had a prior COVID-19 infection.

\section{Methods}

\section{Design, setting and participants}

In a multicenter, prospective, observational pilot study, we tested blood antibody levels to the SARS-CoV-2 Spike (S-protein) and nucleocapsid (N-protein) proteins in hemodialysis, peritoneal dialysis, or healthy control populations (health care workers/staff) at the Diaverum Renal Care 
Centers Potsdam, Ludwigsfelde and Rangsdorf, receiving the BNT162b2/Pfizer-BioNTech (Comirnaty) or ChAdOx1 nCoV-19/Oxford-Astra-Zeneca (Vaxzevria) vaccine, and in hemodialysis patients having previously presented with PCR-positive COVID-19. Approval was obtained from the Ethics Committee of 'Landesärztekammer Brandenburg', Germany (registry number S9/(bB)/2021). The procedures used in this study adhere to the tenets of the Declaration of Helsinki. Written informed consent to participate and publish was obtained from all individual study participants. Information regarding clinical data was collected from medical records. Healthy controls provided demographic data. This manuscript adheres to the 'Strengthening the Reporting of Observational Studies in Epidemiology' guidelines [5].

\section{SARS-CoV-2 antibodies and ACE2-receptor-binding-inhibition capacity}

Participant plasma was collected at baseline and 2 weeks after receiving a first vaccine dose. Participants were tested for SARS-CoV-2 IgG and IgM antibodies directed against the S-protein, and IgG antibodies directed against the SARSCoV-2N-protein. All samples were run on Abbott ARCHITECT $^{\text {TM }}$ i2000SR instrument (Abbott Park, IL). The FDA EUA approved SARS-CoV-2 IgG (List 6R86), AdviseDx SARS-CoV-2 IgM (List 6R87), and SARS-CoV-2 IgG II Quant (List 6S60) assays were used, both automated Chemiluminescent Microparticle Immunoassays (CMIA). Assay results are reported as an index value of the ratio of specimen to calibrator Relative Light Units (RLU) signal. The SARS-CoV-2 IgG II Quant assay is an automated CMIA used for quantitative detection of $\mathrm{IgG}$ antibodies directed against the receptor-binding-domain of the SARS-CoV-2 S-protein. Assay linearity was shown between 21.0 and 40,000 AU/mL. A Research Use Only automated CMIA assay measured the capacity of SARS-CoV-2 antibodies, present in participant plasma, to inhibit SARS-CoV-2 receptor-binding-domain from binding to ACE2-receptors. The laboratory investigators were blinded to the sample sources and clinical outcomes. Researchers who obtained clinical data were blinded to antibody measurements.

\section{Study endpoints}

SARS-CoV-2 spike IgG, IgM and Nucleocapsid IgG levels $(\mathrm{AU} / \mathrm{mL})$ and ACE2-receptor-binding-inhibition capacity (\%) were provided as linear variables. Both response to the first SARS-CoV-2 vaccination and antibody level positivity following prior COVID-19 infection were defined as SARSCoV-2 spike IgG levels $\geq 50 \mathrm{AU} / \mathrm{mL}$.

\section{Statistical analysis}

Study size was determined by the first badge of antibody measurements to gather early potentially important clinical information for this patient population. Values are presented as median (1.-3. quartile). Antibody levels and response status were compared: (i) hemodialysis patients receiving the mRNA-based SARS-CoV-2 vaccination vs. hemodialysis patients receiving the vector-based vaccine, (ii) vaccinated hemodialysis patients versus hemodialysis patients following prior COVID-19 infection, (iii) vaccinated hemodialysis versus peritoneal patients and (iv) vaccinated hemodialysis patients versus staff. Mann-Whitney- $U$-test, $\chi^{2}$ test, or Fisher's exact test were used where appropriate. Alpha was set at 0.05 (2-tailed). SPSS, version 26.0 (IBM Corp., Armonk, NY, USA) was used.

\section{Results}

\section{Participant characteristics}

The 59 participants enrolled in the study included 41 individuals receiving regular hemodialysis, four on peritoneal dialysis, and 14 staff (Fig. 1). Of the hemodialysis patients, 23 received a first dose of the SARS-CoV-2 vaccine (Comirnaty $\mathrm{N}=11$, Vaxzevria $\mathrm{N}=12$ ) and had no recent COVID-19 infection, whereas 18 had a history of COVID-19 but did not receive a SARS-CoV-2 vaccine. Table 1 summarizes the demographic information of vaccinated hemodialysis patients regarding the SARS-CoV-2 vaccine, antibody levels and neutralization capacity before vaccination, previous vaccinations/immunosuppression, dialysis characteristics, comorbidities, medication and routine laboratory values. In other words, a typical cohort with considerable comorbidity and evidence of immunosuppression but with no significant antibody levels against SARS-CoV-2 prior to vaccination.

Peritoneal dialysis patients were aged 60 (52-79) years (all females, one Comirnaty/three Vaxzevria). All staff received Vaxzevria (age 54, [35-56] years, 13 females).

Time from first vaccination to sampling was 14 (13-16) days for hemodialysis patients, 17.5 (13.3-21.0) days for peritoneal dialysis patients and 14 (14-17.5) days for staff.

Time from diagnosis of prior COVID-19 infection to sampling was 6 months $(2.5-12.0)$.

\section{Effect of 1 st vaccination in hemodialysis patients versus peritoneal dialysis patients and staff}

None of the vaccinated hemodialysis or peritoneal patients or staff was positive for the IgG Nucleocapsid-protein, indicating immunity did not result from a recent COVID19 infection. Vaccination responder rates were $17.4 \%$ 
Fig. 1 Patient flow through the study. $H D$, hemodialysis. $P D$, peritoneal dialysis

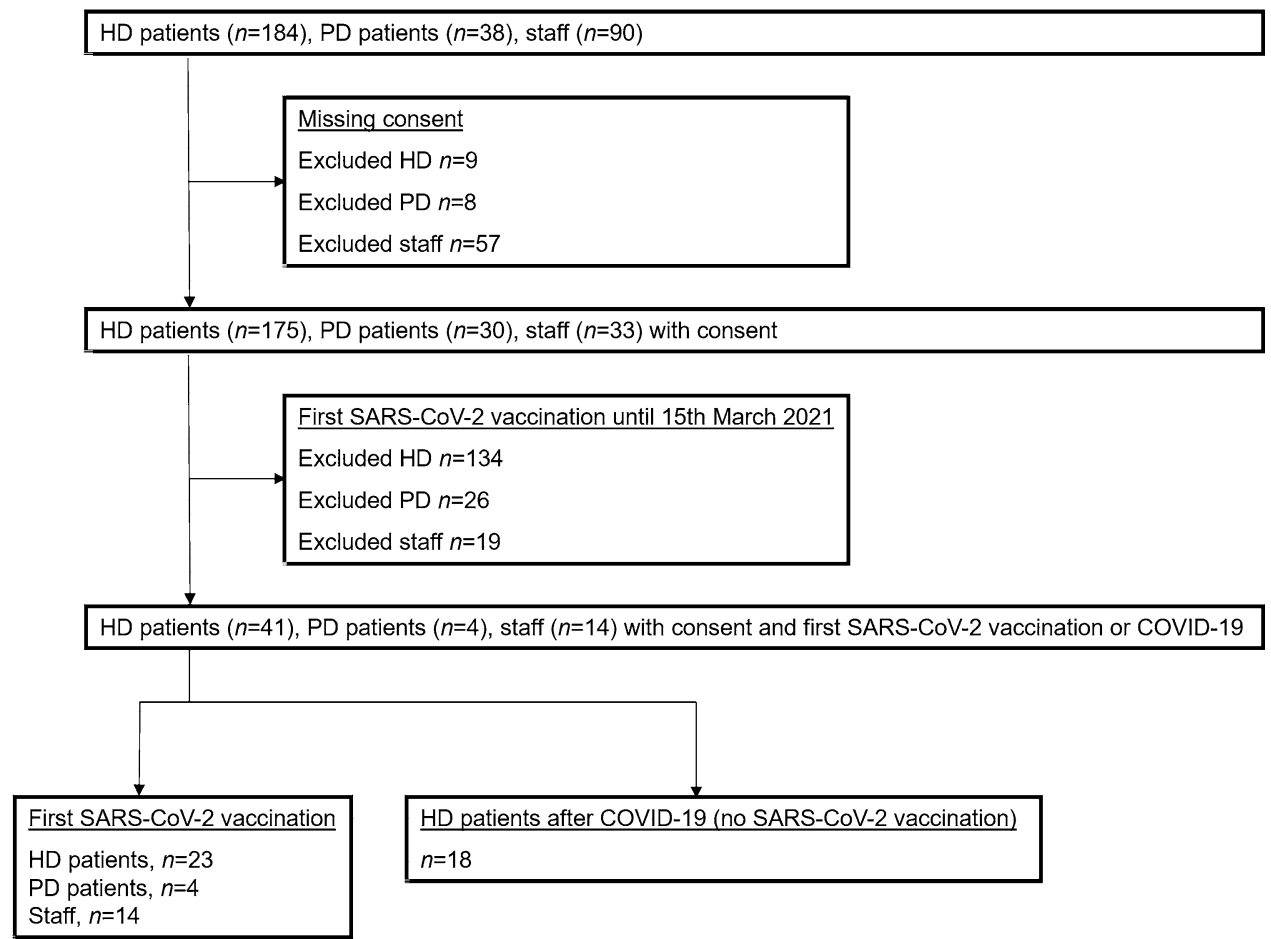

(4/23) in hemodialysis patients, $100 \%$ (4/4) in peritoneal dialysis patients and $57.1 \%(8 / 14)$ in staff (HD vs. PD: $\mathrm{p}=0.004$, HD vs. staff: $\mathrm{p}=0.027)$.

Figure 2 shows lower IgG and IgM spike levels in first vaccinated hemodialysis patients compared to the levels in peritoneal dialysis patients and staff (IgG spike: HD 1.6 [0-14.5] vs. PD 180.7 [82.5-241.9] AU/mL, p=0.011; HD versus staff 73.1 [16.1-1324.5] AU/mL, $\mathrm{p}<0.001$, missing values $\mathrm{N}=0$ ). ACE2-receptor-binding-inhibition capacity was low in vaccinated hemodialysis $(5.0 \%$ [3.1-10.4]) and peritoneal dialysis patients $(12.9 \%$ [9.6-19.8]) and in staff (10.5\% [6.0-40.9]), as well as in hemodialysis patients responding to vaccination $(11.5 \%$ [5.0-27.3]).

\section{Effect of mRNA- versus vector-based SARS-CoV-2 vaccine in hemodialysis patients}

Time from vaccination to sampling was 13.0 (13.0-16.0) days for Comirnaty $(\mathrm{N}=11)$ and 14.5 (14.0-16.8) days for Vaxzevria $(\mathrm{N}=12)$, with two responders to each vaccination. Type of vaccine did not appear to influence the antibody levels of hemodialysis patients (Fig. 3A,B; missing values $\mathrm{N}=0$ ). ACE2-receptor-binding-inhibition was $3.8 \%$ (1.1-11.3) in hemodialysis patients receiving Comirnaty and $7.1 \%(3.8-14.9)$ in those receiving Vaxzevria.

\section{First vaccinated hemodialysis patients versus hemodialysis patients with previous COVID-19 infection}

Of the 18 hemodialysis patients with prior COVID-19 infection, 15 were positive for the IgG Nucleocapsid-protein. IgM spike levels were $0.86(0.03-7.46)$ and Nucleocapsid-protein Index levels were 3.0 (1.4-6.0).

Of the hemodialysis patients with prior COVID-19 infection, those with IgG spike-positivity $(13 / 18,72.2 \%)$ had higher IgG spike levels and ACE2-receptor-bindinginhibition capacity compared with that of first dose vaccination-responding hemodialysis patients $(\mathrm{N}=4 / 23)$ : $\mathrm{IgG}$ spike: 3,794.6 (793.4-9357.9) vs. 222.0 (104.1-721.9) AU/mL, $\mathrm{p}=0.015$; ACE2-receptor-binding-inhibition capacity: $74.8 \%(44.9-98.1)$ vs. $11.5 \%$ (5.0-27.3), $\mathrm{p}=0.002$.

\section{SARS-CoV-2 IgG spike levels and ACE2-receptor-binding-inhibition capacity}

There was high correlation between reported IgG spike levels and ACE2-receptor-binding-inhibition capacity (Spearman correlation coefficient $r=0.89, \mathrm{p}<0.001$ ). 
Table 1 Baseline characteristics

\begin{tabular}{|c|c|}
\hline & $\begin{array}{l}\text { Hemodialysis patients after } 1 \text { st mRNA- } \\
\text { or vector-based SARS-CoV-2 vaccina- } \\
\text { tion } \\
\mathrm{N}=23\end{array}$ \\
\hline Age & $64(61-83)$ \\
\hline Female & $8(34.8 \%)$ \\
\hline Vintage (months) & $26(13-50)$ \\
\hline Body mass index $\left(\mathrm{kg} / \mathrm{m}^{2}\right)$ & $27.5(25.3-30.8)$ \\
\hline Nursing home & $2(8.7 \%)$ \\
\hline Disability & $4(17.4 \%)$ \\
\hline Tobacco use & $1(4.4 \%)$ \\
\hline Alcohol abuse disorder & $3(13.0 \%)$ \\
\hline Drug abuse disorder & $0(0 \%)$ \\
\hline \multicolumn{2}{|l|}{ SARS-CoV-2 vaccination-related information } \\
\hline 1st SARS-CoV-2 vaccination (comirnaty/vaxzevria) & $11(47.8 \%) / 12(52.2 \%)$ \\
\hline Interval between 1st SARS-CoV-2 vaccination and sampling, days & $14(13-16)$ \\
\hline Hospitalization within 14 days after 1 st SARS-CoV-2 vaccination & $0(0 \%)$ \\
\hline \multicolumn{2}{|l|}{ Antibody levels and neutralization capacity before vaccination ${ }^{a}$} \\
\hline IgG spike, $\mathrm{AU} / \mathrm{mL}$ & $0.0(0.0-0.8)$ \\
\hline IgM spike, index & $0.03(0.02-0.04)$ \\
\hline IgG nucleocapsid, index & $0.05(0.02-0.08)$ \\
\hline ACE2 receptor binding inhibition, $\%$ & $4.4(3.1-5.9)$ \\
\hline \multicolumn{2}{|l|}{ Previous vaccinations or immunosuppression } \\
\hline Other vaccines within 14 days & $0(0 \%)$ \\
\hline Time from previous vaccination to SARS-CoV-2 vaccination, months & $4.5(2.8-5.0)$ \\
\hline Potential immunosuppression & $3(13.0 \%)$ \\
\hline History of kidney transplantation & $5(21.7 \%)$ \\
\hline Immunodeficiency disorder (other than kidney transplantation) & $3(13.0 \%)$ \\
\hline \multicolumn{2}{|l|}{ Dialysis-related information } \\
\hline Charlson comorbidity index & $4.0(3.0-5.5)$ \\
\hline Diabetic nephropathy & $3(13.0 \%)$ \\
\hline Hypertensive kidney disease & $12(52.2 \%)$ \\
\hline Glomerulonephritis & $2(8.7 \%)$ \\
\hline Autosomal dominant polycystic kidney disease & $2(8.7 \%)$ \\
\hline Other/unknown primary kidney disease & $4(17.4 \%)$ \\
\hline $\mathrm{Kt} / \mathrm{V}$ & $1.8(1.5-2.0)$ \\
\hline Fistula & $17(73.9 \%)$ \\
\hline Graft & $3(13.0 \%)$ \\
\hline Central venous catheter & $3(13.0 \%)$ \\
\hline \multicolumn{2}{|l|}{ Comorbidities } \\
\hline Number of comorbidities & $16(13-21)$ \\
\hline Transplantation candidate & $13(56.5 \%)$ \\
\hline Obesity (body mass index > 30) & $2(8.7 \%)$ \\
\hline Diabetes mellitus & $6(26.1 \%)$ \\
\hline Hypertension & $22(95.7 \%)$ \\
\hline Ischemic heart disease & $8(34.8 \%)$ \\
\hline Congestive heart failure & $8(34.8 \%)$ \\
\hline Chronic obstructive disease & $4(17.4 \%)$ \\
\hline Stroke/cerebrovascular disorder & $3(13.0 \%)$ \\
\hline Peripheral vascular disease & $2(8.7 \%)$ \\
\hline History of malignancy & $7(30.4 \%)$ \\
\hline
\end{tabular}


Table 1 (continued)

\begin{tabular}{|c|c|}
\hline & $\begin{array}{l}\text { Hemodialysis patients after } 1 \text { st mRNA- } \\
\text { or vector-based SARS-CoV-2 vaccina- } \\
\text { tion } \\
\mathrm{N}=23\end{array}$ \\
\hline Thyroid disorder & $8(34.8 \%)$ \\
\hline \multicolumn{2}{|l|}{ Medications } \\
\hline Erythropoiesis stimulating agents dose (unit per week) & $4,000(550-10,000)$ \\
\hline Iron dose (mg/week) & $40(10-50)$ \\
\hline Angiotensin blockers & $12(52.2 \%)$ \\
\hline ACE inhibitors & $4(17.4 \%)$ \\
\hline Betablockers & $19(82.6 \%)$ \\
\hline Calcium antagonists & $12(52.2 \%)$ \\
\hline Diuretics & $14(60.9 \%)$ \\
\hline Phosphate binders & $13(56.5 \%)$ \\
\hline Insulin & $4(17.4 \%)$ \\
\hline Vitamin D & $17(73.9 \%)$ \\
\hline Active vitamin D & $14(60.9 \%)$ \\
\hline \multicolumn{2}{|l|}{ Laboratory values } \\
\hline Albumin $\mathrm{g} / \mathrm{L}$ & $37(33-41)$ \\
\hline Hypoalbuminemia (albumin $<3.5 \mathrm{~g} / \mathrm{L}$ ) & $10(43.5 \%)$ \\
\hline Hemoglobin (g/dL) & $11.1(10.7-11.4)$ \\
\hline Transferrin saturation $(\%)$ & $25.0(19.7-29.0)$ \\
\hline Ferritin $(\mathrm{mg} / \mathrm{dL})$ & $422(271-484)$ \\
\hline White blood cell count & $5.9(5.4-7.2)$ \\
\hline C-reactive protein, $\mathrm{ml} / \mathrm{L}$ & $4(2-10)$ \\
\hline
\end{tabular}

SARS-CoV-2, severe acute respiratory syndrome coronavirus 2

${ }^{\text {a }}$ Missing values $\mathrm{N}=14$

\section{Discussion}

Two weeks after the first SARS-CoV-2 vaccine dose, we demonstrated a low responder rate and minimal neutralizing antibody levels in hemodialysis patients regardless of the type of vaccine. Following prior COVID-19 infection in hemodialysis patients, antibody-related immunity was more pronounced than that of responding first vaccinated hemodialysis patients. There was a strong correlation between SARS-CoV-2 IgG spike levels and ACE2-receptor-bindinginhibition capacity.

Notably, COVID-19 occurring in the normal population shortly after first vaccination has been described. A recent study reported that, as vaccination programs start to roll out, social distancing decreases due to the anticipated efficacy of SARS-CoV-2 vaccinations [6]. Recently, reduced antibody response after the first dose of mRNA-based COVID-19 vaccine in hemodialysis patients was briefly reported [7]. However, the effect of the first dose of a vector-based vaccine in hemodialysis patients remains unknown and peritoneal dialysis patients have not yet been investigated in this regard.

The findings of the present study are novel regarding the severely impaired quantitative and qualitative antibody-related response in hemodialysis patients 2 weeks after the first dose of both mRNA- and vector-based vaccines. To prevent new cases of COVID-19 between the first and second vaccination, our study findings suggest that SARS-CoV-2 protective measures should at least be sustained in dialysis patients and staff until the full effect of the second vaccination dose is achieved. Study results also imply that hemodialysis patients should not be considered for delayed second dose of vaccination. Our study might point toward more rapid vaccination response in peritoneal dialysis patients. The high antibody levels in patients with prior COVID-19 infection confirms previous findings in patients on maintenance dialysis who recovered from COVID-19 [8]. Whether this persistent immunity may predispose dialysis patients to a similar triggering effect of a single vaccine dose as described for other populations, remains to be studied [9]. The demonstrated strong association between IgG spike levels and ACE2receptor-binding-inhibition elicited by a single vaccine dose in dialysis patients was previously shown in patients with COVID-19 [10] and may confirm assay usability in dialysis patients. 
Fig. 2 SARS-CoV-2 IgG (A) and $\operatorname{IgM}(\mathbf{B})$ spike levels in HD and PD patients and staff 2 weeks after first vaccination and in HD patients after COVID-19 infection. A Median (1;3 quartile). HD patients 2 weeks after first vaccination $(\mathrm{N}=23)$ : $1.6(0-14.5)$ AU/ $\mathrm{mL}$. PD patients 2 weeks after first vaccination $(\mathrm{N}=4)$ : 180.7 (82.5-241.9) AU/mL. Staff 2 weeks after first vaccination ( $\mathrm{N}=14)$ : 73.1 (16.1-1324.5) AU/mL. HD patients after COVID-19 ( $\mathrm{N}=18)$ : 818.4 (1.6-7806.1) AU/mL. B Median (1;3 quartile). HD patients 2 weeks after first vaccination ( $\mathrm{N}=23)$ : 0.04 (0.03-0.21) Index. PD patients 2 weeks after first vaccination $(\mathrm{N}=4)$ : 0.08 (0.07-0.32) Index. Staff 2 weeks after first vaccination $(\mathrm{N}=14)$ : 0.34 (0.22-1.67) Index. HD patients after COVID-19 $(\mathrm{N}=18)$ : $0.86(0.03-7.46)$ Index. Missing values IgG and IgM spike: $\mathrm{N}=0$. $H D$, hemodialysis. $P D$, peritoneal dialysis
A

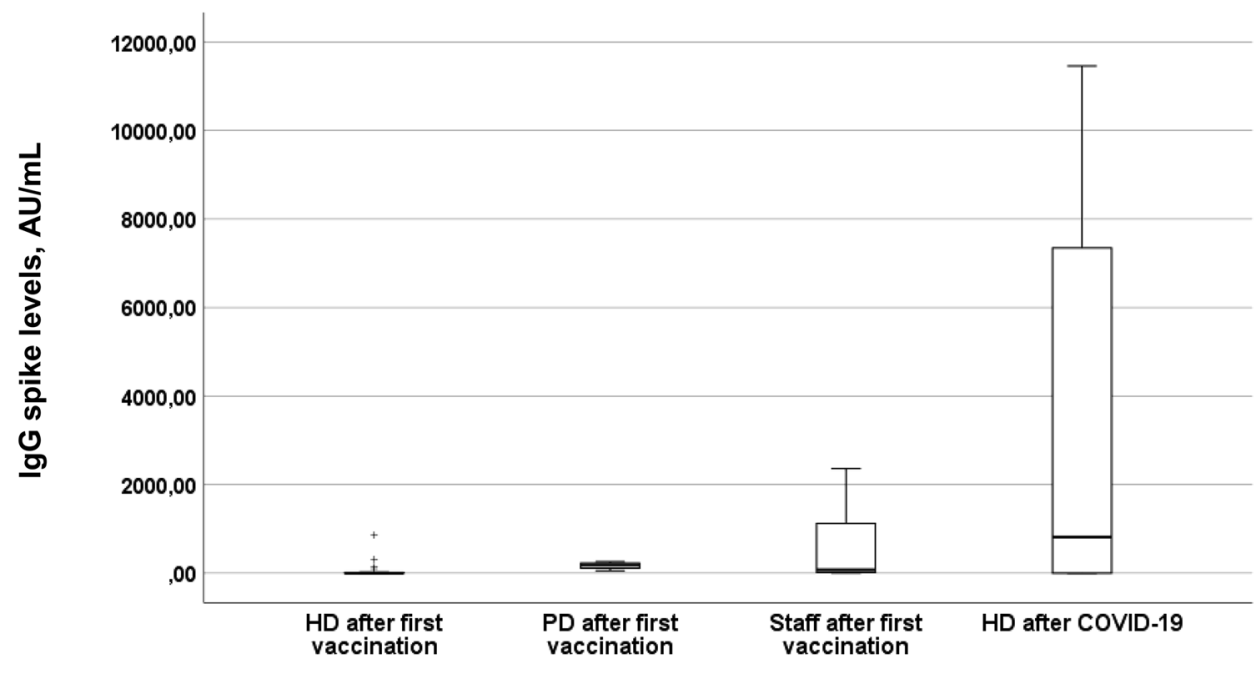

B

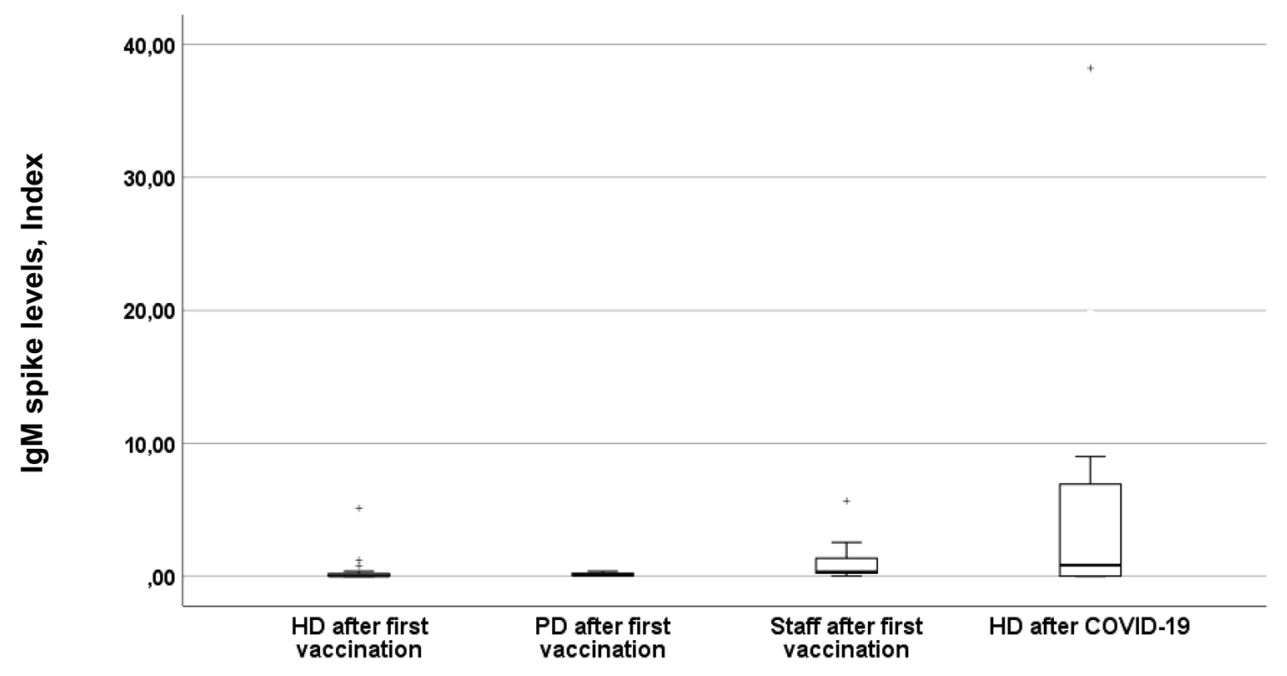

\section{Strengths and limitations}

The generalizability of our study results is limited by the small patient number. Cell-related immunity was not measured. However, we report the findings of a prospective multicenter pilot study including results of a neutralizing antibody assay and have taken advantage of the use of different types of vaccinations in our hemodialysis patients. Furthermore, healthy staff after first vaccination dose and hemodialysis patients after COVID-19 infection were reported as control groups.
In conclusion, most hemodialysis patients and a considerable proportion of healthy staff exhibited an insufficient antibody-related response 2 weeks after a first mRNA- or vector-based SARS-CoV-2 vaccination, indicating the need to continue with protective measures. Patients with prior COVID-19 infection demonstrated a persistence of 
Fig. 3 Response of HD patients to different types of vaccinations-SARS-CoV-2 IgG (A) and $\operatorname{IgM}$ (B) spike levels 2 weeks after first vaccination. A IgG Median (1;3 quartile): Comirnaty $(\mathrm{N}=11): 0.0$ (0.0-3.8) AU/mL. Vaxzevria $(\mathrm{N}=12): 4.3(1.6-20.1) \mathrm{AU} /$ $\mathrm{mL} \cdot \mathrm{p}=0.079$. B IgM Median (1;3 quartile): Comirnaty $(\mathrm{N}=11): 0.04(0.03-0.39)$ Index. Vaxzevria $(\mathrm{N}=12): 0.04$ (0.02-0.17) Index. $\mathrm{p}=0.786$. Missing values IgG and IgM spike: $\mathrm{N}=0, H D$, hemodialysis

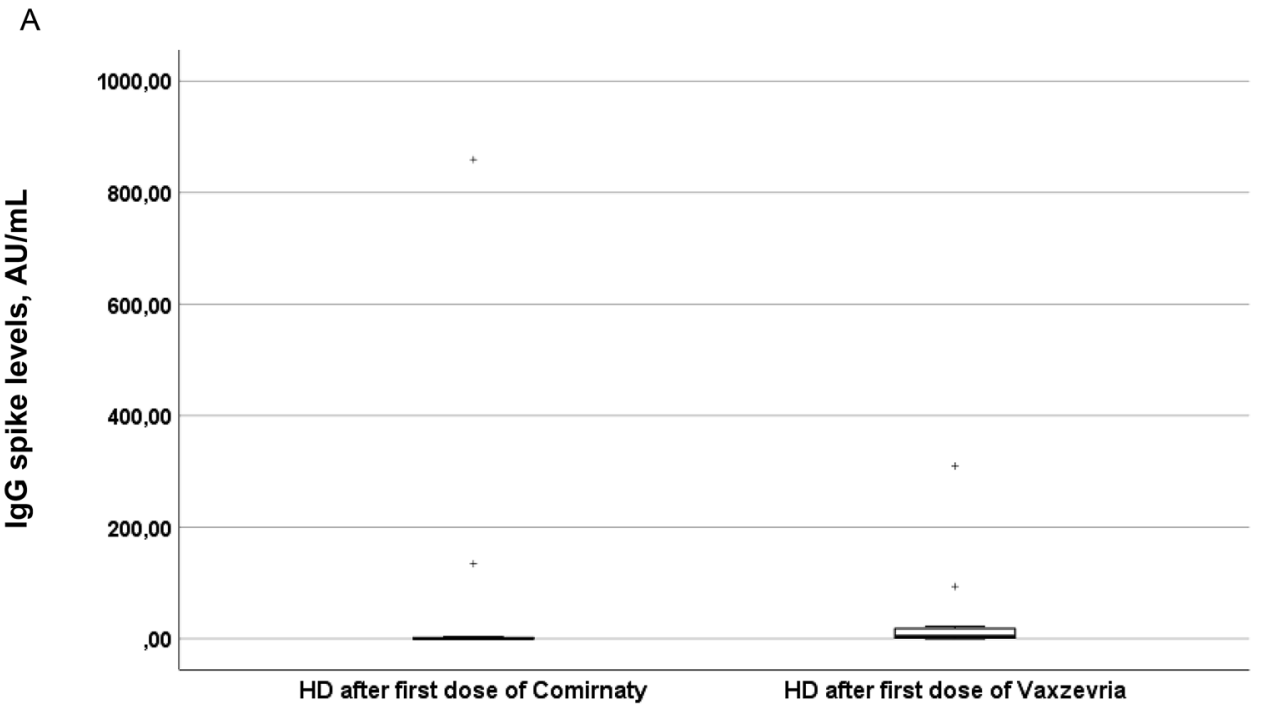

B

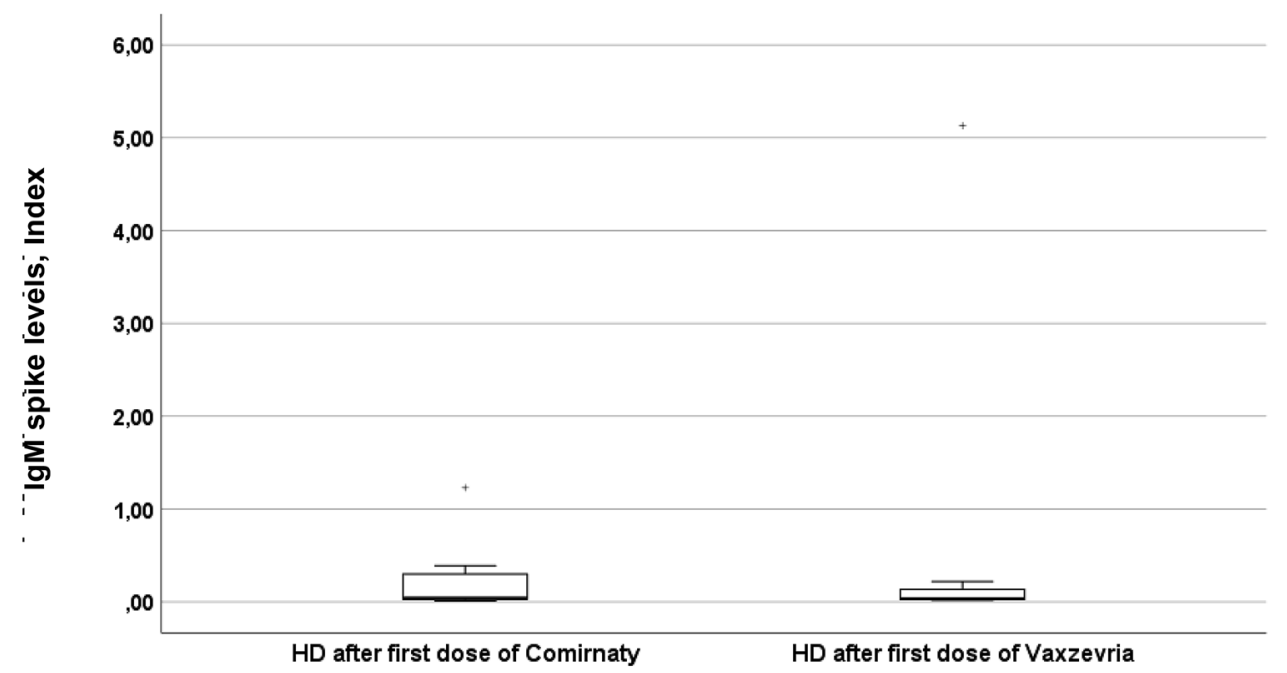

SARS-CoV-2 spike-protein antibodies. Further studies with serial measurements of spike-protein antibodies are needed to determine whether this antibody persistence may result in a triggering effect of a single vaccine dose, thus potentially saving the need for a second dose in hemodialysis patients with prior COVID-19.

Acknowledgements We thank the staff of the Diaverum Renal Care Centers Potsdam, Ludwigsfelde and Rangsdorf for their excellent assistance.

Author contributions Conceptualization: PL, MH; Methodology: PL, MH; Formal analysis and investigation: MH, PL, MA, GC, MS, AH, MH, CS, CL, FM; Writing - original draft preparation: MH, AH; Writing-review and editing: $\mathrm{PL}, \mathrm{MH}, \mathrm{AH}, \mathrm{MA}, \mathrm{GC}, \mathrm{MS}, \mathrm{MH}, \mathrm{CS}, \mathrm{CL}$,
FM; Funding acquisition: MH, AH; Resources: PL, MH, AH; Supervision: PL, MH.

Funding Open Access funding enabled and organized by Projekt DEAL. Antibodies were measured by Abbott Laboratories and supported by Abbott's internal Research and Development funding.

Availability of data and materials The datasets generated and/or analyzed during the current study are available from the corresponding author on reasonable request.

\section{Declarations}

Conflict of interest Mark Anderson, Gavin Cloherty, Michael Stec: Employee of Abbott Laboratories. The other authors declare no conflict of interest. 
Ethics approval The study was approved by the Ethics Committee of the 'Landesärztekammer Brandenburg', Germany (registry number S $9 /(\mathrm{bB}) / 2021)$. The procedures used in this study adhere to the tenets of the Declaration of Helsinki. Written informed consent to participate and to publish was obtained from all individual participants included in the study.

Consent to participate Written informed consent to participate was obtained from all individual participants included in the study.

Consent for publication Written informed consent to publish was obtained from all individual participants included in the study.

Open Access This article is licensed under a Creative Commons Attribution 4.0 International License, which permits use, sharing, adaptation, distribution and reproduction in any medium or format, as long as you give appropriate credit to the original author(s) and the source, provide a link to the Creative Commons licence, and indicate if changes were made. The images or other third party material in this article are included in the article's Creative Commons licence, unless indicated otherwise in a credit line to the material. If material is not included in the article's Creative Commons licence and your intended use is not permitted by statutory regulation or exceeds the permitted use, you will need to obtain permission directly from the copyright holder. To view a copy of this licence, visit http://creativecommons.org/licenses/by/4.0/.

\section{References}

1. Quintaliani G, Reboldi G, Di Napoli A et al (2020) Exposure to novel coronavirus in patients on renal replacement therapy during the exponential phase of COVID-19 pandemic: survey of the Italian Society of Nephrology. J Nephrol 33:725-736. https://doi. org/10.1007/s40620-020-00794-1

2. Haarhaus M, Santos C, Haase M et al (2021) Risk prediction of COVID-19 incidence and mortality in a large multi-national hemodialysis cohort: implications for management of the pandemic in outpatient hemodialysis settings. Clin Kidney J 14:805-813. https://doi.org/10.1093/ckj/sfab037

3. Schrezenmeier E, Bergfeld L, Hillus D et al (2021) Immunogenicity of COVID-19 tozinameran vaccination in patients on chronic dialysis. medRxiv. https://doi.org/10.1101/2021.03.31.21254683

4. Grupper A, Sharon N, Finn T et al (2021) Humoral response to the Pfizer BNT162b2 vaccine in patients undergoing maintenance hemodialysis. Clin J Am Soc Nephrol. https://doi.org/10.2215/ CJN.03500321

5. von Elm E, Altman DG, Egger M, Pocock SJ, Gotzsche PC, Vandenbroucke JP (2014) The Strengthening the Reporting of Observational Studies in Epidemiology (STROBE) statement: guidelines for reporting observational studies. Int J Surg 12:1495-1499. https://doi.org/10.1016/j.ijsu.2014.07.013

6. Andersson O, Campos-Mercade P, Meier A, Wengström E (2020) Anticipation of COVID-19 vaccines reduces social distancing. SSRN. https://doi.org/10.2139/ssrn.3765329

7. Torreggiani M, Blanchi S, Fois A, Fessi H, Piccoli GB (2021) Neutralizing SARS-CoV-2 antibody response in dialysis patients after the first dose of the BNT162b2 mRNA COVID-19 vaccine: the war is far from being won. Kidney Int. https://doi.org/10. 1016/j.kint.2021.04.010

8. Ikizler TA, Coates PT, Rovin BH, Ronco P (2021) Immune response to SARS-CoV-2 infection and vaccination in patients receiving kidney replacement therapy. Kidney Int. https://doi.org/ 10.1016/j.kint.2021.04.007

9. Levi R, Azzolini E, Pozzi C et al (2021) One dose of SARS-CoV-2 vaccine exponentially increases antibodies in recovered individuals with symptomatic COVID-19. J Clin Investig. https://doi.org/ 10.1172/JCI149154

10. Ebinger JE, Fert-Bober J, Printsev I et al (2021) Antibody responses to the BNT162b2 mRNA vaccine in individuals previously infected with SARS-CoV-2. Nat Med. https://doi.org/10. 1038/s41591-021-01325-6

Publisher's Note Springer Nature remains neutral with regard to jurisdictional claims in published maps and institutional affiliations. 